\title{
Pulsating Variable Stars in Local Group Dwarf Galaxies
}

\author{
Mario Mateo \\ Department of Astronomy, University of Michigan, 821 Dennison Bldg., \\ Ann Arbor, MI 48109-1090, USA
}

\begin{abstract}
Pulsating variables stars have had a long and prominent history in helping to map out the local Universe. Current observational techniques now make it possible to identify examples of all non-degenerate radial pulsating variables throughout much of the Local Group. I review our current knowledge of such variables in the dwarf galaxies of the Local Group. This summary tries to highlight some recent results that help underscore the use of these stars as important tools to study the physical nature of their parent galaxies as well as their continued use as important distance indicators of nearby galaxies.
\end{abstract}

\section{Introduction}

Hubble (1925) opened the door to extragalactic astronomy with his discovery and analysis of Cepheids in NGC 6822, a relatively little-known dwarf galaxy of the Local Group (LG). Hubble had good reasons to start his landmark studies with a dwarf system. First, he was able to conveniently survey virtually the entire galaxy with available plate materials and telescopes. Second, Hubble understood that the Cepheid populations varied systematically within the much larger galaxy M31. This demanded a larger database to obtain a representative sample of Cepheids and meant that the basic properties of these stars perhaps even their underlying period-luminosity relation - may vary within the giant system. NGC 6822 offered a more homogeneous sample of Cepheids for initial study. Third, Hubble understood that differential extinction was severely present in M31, but much reduced in NGC 6822. Finally, the lower stellar density within the dwarf galaxy made his search for faint variables considerably more productive, especially with the earliest plate materials.

Over the years, our knowledge of pulsating variables and of the galaxies of the Local Group has grown tremendously. Table 1 summarizes the dwarf membership within the LG and provides a census - meant to be precise as of August 1999 - of known pulsating variables in each galaxy. I do not include data for the numbers of variables in M31, the Milky Way, M33, and the Magellanic Clouds, principally because of the dizzying rate at which our knowledge of variables is growing in these systems and also because many papers in this volume address this in more detail and authority than I could here.

Recent reviews cover the basic properties of the dwarf galaxies listed in Table 1 (e.g. Grebel 1997, Mateo 1998; van den Bergh 1999), but I wish to stress a few of those properties here. First, as Hubble understood for NGC 6822 , 
the dwarfs tend to exhibit fairly homogeneous populations. Certainly, the inhomogeneities within individual galaxies have become an increasingly active area of study (Hurley-Keller, Mateo, \& Nemec 1998, Hurley-Keller, Mateo, \& Keller 1999), but compared to giant systems such as the Milky Way and M31, these smaller systems contain stellar populations of nearly constant metallicity (or metallicity range) with relatively modest spatial variations in the mixture of stellar ages. In practice, the LG dwarfs are dominated by intermediate-age and old stars, hence variables associated with Population II stars are well represented (see Nemec, Nemec, \& Lutz 1994 for an excellent review of the global properties of these stars). Second, the dwarfs are not small systems in the sky; typical systems cover $0.25-1$ square degree. Thus, comprehensive and complete surveys of their variable stars demand efforts comparable to those of modern microlensing surveys.

\section{The Anomaly of Anomalous Cepheids}

Figure 6 of Mateo, Fischer, \& Krzeminski (1995) presents a color-magnitude diagram of the Sextans dwarf spheroidal galaxy showing the locations of all bright variable stars. The objects that lie above the obvious RR Lyrae stars of the galaxy's horizontal branch are known as anomalous Cepheids (AC). These stars have luminosities that range from about $50 \%$ to 4 times higher than those of RR Lyrae stars, and exhibit periods ranging from 0.3 to $3 \mathrm{~d}$ or longer. The short-period ACs are particularly troublesome since they mimic RR Lyrae stars quite effectively; only in systems where the variables are at a common distance, such as dwarfs, can one unambiguously distinguish these stars. This may be why only one $\mathrm{AC}$ is known to exist within the Milky Way (in the globular cluster NGC 5466), though many may be hidden among the large samples of field RR Lyrae stars. In contrast, all of the dwarf spheroidal galaxies are known to contain these stars.

Models by Bono et al. (1997) suggest that ACs require (a) relatively high masses $\left(\sim 1.2-1.8 M_{0}\right)$ compared to ancient stellar populations, and (b) low metallicities to reach the instability strip during their blue-loop excursions of the core He burning evolutionary stage. Because most dwarf galaxies are metal poor systems (Skillman, Kennicutt, \& Hodge 1989) and because these systems contain a range of stellar ages (Mateo 1998), both conditions can be met and $\mathrm{ACs}$ are favored to form in these environments. Mateo et al. (1995) noted the surprising result that the luminosity-normalized frequency of ACs in dSph galaxies was proportional to luminosity. That is, the total numbers of ACs is approximately constant (about 10) regardless of galaxy luminosity. Given the apparently random distribution of star-formation histories in these systems, this result remains surprising. More complete surveys of ACs in all the nearby dwarf galaxies may shed some light on this issue.

\section{RR Lyrae Stars: Beacons of Ancient Populations}

Although recent advances in detectors have greatly improved the ability to carry out faint photometry at or near the photon-statistical limit, it remains difficult to identify the turnoff corresponding to the oldest stars in all but the closest 
Table 1. Radially Pulsating Stars in Local Group Dwarfs

\begin{tabular}{|c|c|c|c|c|c|c|c|c|}
\hline Galaxy & $\begin{array}{c}\text { Distance } \\
\mathrm{kpc}\end{array}$ & $\begin{array}{c}L \\
10^{6} L_{\odot}\end{array}$ & $\overline{[\mathrm{Fe} / \mathrm{H}]}$ & $N_{\text {Ceph }}$ & $N_{\mathrm{AC}}$ & $N_{\mathrm{RR}}$ & $N_{\mathrm{DC}}$ & $N_{\mathrm{LPV}}$ \\
\hline WLM & 925 & 500 & -1.5 & 15 & $\mathrm{xxx}$ & $\mathrm{xxx}$ & - & $\mathrm{xxx}$ \\
\hline M32 & 805 & 380 & -1.1 & - & $\mathrm{xxx}$ & $\mathrm{xxx}$ & - & $\mathrm{xxx}$ \\
\hline NGC 205 & 815 & 370 & -0.8 & - & $\sim 9$ & 30 & - & 12 \\
\hline NGC 3109 & 1250 & 160 & -1.5 & 29 & $\mathrm{xxx}$ & - & - & $\mathrm{xxx}$ \\
\hline IC 10 & 825 & 160 & -1.1 & $5-9$ & $\mathrm{xxx}$ & - & - & $\mathrm{xxx}$ \\
\hline NGC 185 & 620 & 130 & -1.2 & - & - & 151 & - & $\mathrm{xxx}$ \\
\hline NGC 147 & 725 & 130 & -1.1 & - & - & 32 & - & $\mathrm{xxx}$ \\
\hline NGC 6822 & 490 & 94 & -1.2 & 13 & $\mathrm{xxx}$ & $\mathrm{xxx}$ & - & $\mathrm{xxx}$ \\
\hline IC 5152 & 1590 & 70 & -0.9 & Yes? & - & - & - & $\mathrm{xxx}$ \\
\hline IC 1613 & 700 & 64 & -1.3 & $77+$ & - & 15 & - & $\mathrm{xxx}$ \\
\hline Sextans A & 1440 & 56 & -1.9 & 10 & $\mathrm{xxx}$ & - & - & $\mathrm{xxx}$ \\
\hline Sextans B & 1345 & 41 & -1.2 & 7 & 6 & 36 & - & 1 \\
\hline Sagittarius & 24 & 18 & -1.0 & - & 4 & $313+$ & Yes & $\mathrm{xxx}$ \\
\hline Fornax & 138 & 16 & -1.3 & 1 & 1 & $400+$ & Yes? & 30 \\
\hline Pegasus & 955 & 12 & -1.0 & $7-10$ & $\mathrm{xxx}$ & $\mathrm{xxx}$ & - & $\mathrm{xxx}$ \\
\hline EGB $0427+63$ & 1300 & 9.1 & -1.7 & - & - & - & - & $\mathrm{xxx}$ \\
\hline SagDIG & 1060 & 6.9 & -1.9 & - & - & - & - & $\mathrm{xxx}$ \\
\hline And VII & 770 & 5.7 & -1.4 & - & $\mathrm{xxx}$ & $\mathrm{xxx}$ & - & $\mathrm{xxx}$ \\
\hline UKS2323-326 & 1320 & 5.3 & - & - & - & - & - & $\mathrm{xxx}$ \\
\hline Leo I & 250 & 4.8 & -1.5 & - & 15 & Yes? & $\mathrm{xxx}$ & $\mathrm{xxx}$ \\
\hline And I & 805 & 4.7 & -1.5 & - & $\mathrm{xxx}$ & $4+$ & - & $\mathrm{xxx}$ \\
\hline Leo A & 690 & 3.0 & -2.0 & $5 ?$ & $\mathrm{xxx}$ & $\mathrm{xxx}$ & - & $\mathrm{xxx}$ \\
\hline And II & 525 & 2.4 & -1.6 & - & $\mathrm{xxx}$ & $\mathrm{xxx}$ & - & $\mathrm{xxx}$ \\
\hline Sculptor & 79 & 2.2 & -1.8 & - & $11 ?$ & $226+$ & $40+$ & 1 \\
\hline Antlia & 1235 & 1.7 & -1.8 & - & - & $\mathrm{xxx}$ & - & $\mathrm{xxx}$ \\
\hline And VI & 830 & 1.4 & -1.5 & - & $\mathrm{xxx}$ & $\mathrm{xxx}$ & - & $\mathrm{xxx}$ \\
\hline LGS 3 & 810 & 1.3 & -1.8 & - & $\mathrm{xxx}$ & $\mathrm{xxx}$ & - & $\mathrm{xxx}$ \\
\hline And III & 760 & 1.1 & -2.0 & - & $\mathrm{xxx}$ & $\mathrm{xxx}$ & - & $\mathrm{xxx}$ \\
\hline And V & 800 & 1.0 & -1.5 & - & $\mathrm{xxx}$ & $\mathrm{xxx}$ & - & $\mathrm{xxx}$ \\
\hline Phoenix & 445 & 0.9 & -1.9 & $60+$ & $\mathrm{xxx}$ & $\mathrm{xxx}$ & - & $\mathrm{xxx}$ \\
\hline DDO 210 & 800 & 0.8 & -1.5 & - & - & - & - & $\mathrm{xxx}$ \\
\hline Tucana & 880 & 0.6 & -1.7 & - & Yes & - & - & $3 ?$ \\
\hline Leo II & 205 & 0.6 & -1.9 & 0 & 4 & 152 & $\mathrm{xxx}$ & $\mathrm{xxx}$ \\
\hline Cetus & 800 & 0.6 & - & - & $\mathrm{xxx}$ & $\mathrm{xxx}$ & - & $\mathrm{xxx}$ \\
\hline Sextans & 86 & 0.5 & -1.7 & - & 6 & 36 & $\mathrm{xxx}$ & 1 \\
\hline Carina & 101 & 0.4 & -2.0 & - & 9 & 69 & 20 & $\mathrm{xxx}$ \\
\hline Ursa Minor & 66 & 0.3 & -2.2 & - & 7 & 82 & $\mathrm{xxx}$ & $\mathrm{xxx}$ \\
\hline Draco & 82 & 0.3 & -2.0 & - & $5+$ & $280+$ & $\mathrm{xxx}$ & $\mathrm{xxx}$ \\
\hline
\end{tabular}

Entries with ' $x x x$ ' refer to cases where searches could be carried out but no suitable data exist yet. 
external galaxies. RR Lyrae stars represent much more easily identified beacons that signal the presence of an ancient population. How ancient? Olszewski, Suntzeff, \& Mateo (1996) noted that the clusters of the Magellanic Clouds can be used to help estimate the youngest age population in which RR Lyrae stars can form; though the procedure remains uncertain because of the sparseness of some of the critical clusters, it appears that only populations older than about $10 \mathrm{Gyr}$ will contain these variables. Thus, the mere presence of RR Lyrae stars (Table 1) is indicative of an ancient stellar component; in many of the cases listed in the table (and many that are not listed for galaxies in more distant groups), the RR Lyrae stars tell us that old populations exist even where it remains impossible to identify the ancient main-sequence turnoff directly.

The narrow luminosity range for $R R$ Lyrae stars also makes them a useful tool to study the three-dimensional structures of nearby systems. One particularly useful case is the Sgr dwarf galaxy (Ibata, Gilmore, \& Irwin 1994), the closest external galaxy to the Milky Way. This system is also being severely disrupted by its tidal interaction with our Galaxy. Models broadly predict that the encounter is shredding Sgr and that dwarf may exhibit considerable depth along certain lines of sight. Mateo et al. (1995) and Alard (1996) found RR Lyrae stars in Sgr soon after its discovery; the total population may exceed 1500 variables. Lepri et al. (1997) have reported initial results of a study to use these stars to determine the orientation and shape of Sgr perpendicular to the plane of the sky by measuring systematic variations in the mean luminosities of RR Lyrae stars in different locations of the system. In a similar way, RR Lyrae stars may prove useful to identify substructure in the Galactic halo and possibly in the future - using the enormous RR. Lyrae samples from microlensing surveys - to better trace the depth of the LMC and particularly the more-disturbed SMC.

\section{Dwarf Cepheids: Pushing the Envelope}

The classical instability strip extends below the horizontal branch and intersects the main sequence at $M_{V} \sim+2.5 \pm 1.0$. In this region, so-called dwarf Cepheids (DCs) can be found if main sequence stars extend to this luminosity. Some globular clusters with blue stragglers in fact contain dwarf Cepheids, and, it turns out, dSph galaxies with intermediate-age populations do too. The first such extragalactic DCs were identified by Mateo, Hurley-Keller, \& Nemec (1998; see also Poretti 1999) in the Carina dwarf galaxy. As summarized by Nemec et al. (1994) and McNamara (1997), these pulsators obey a well-defined periodluminosity relation; the slope of the relation in Carina matches that found from field DCs and offers a new way to estimate distances to galaxies containing these stars. This is potentially a very valuable feature since some DCs have reasonably well-determined parallaxes from Hipparcos. In this way, the distance scale from the DCs can be used to help (re-)establish the RR Lyrae scale. Such cross-ties between distance scale for different pulsators are crucial; after all, the distances determined from any one of these must ultimately agree with those from all the others if we are to claim we have properly calibrated the various scales.

In practice, $D C s$ are difficult to identify and to study in even the nearest dwarfs because (a) they are intrinsically low-luminosity stars compared to the other sorts of Pop II pulsators (Nemec et al. 1994), and (b) their pulsation peri- 


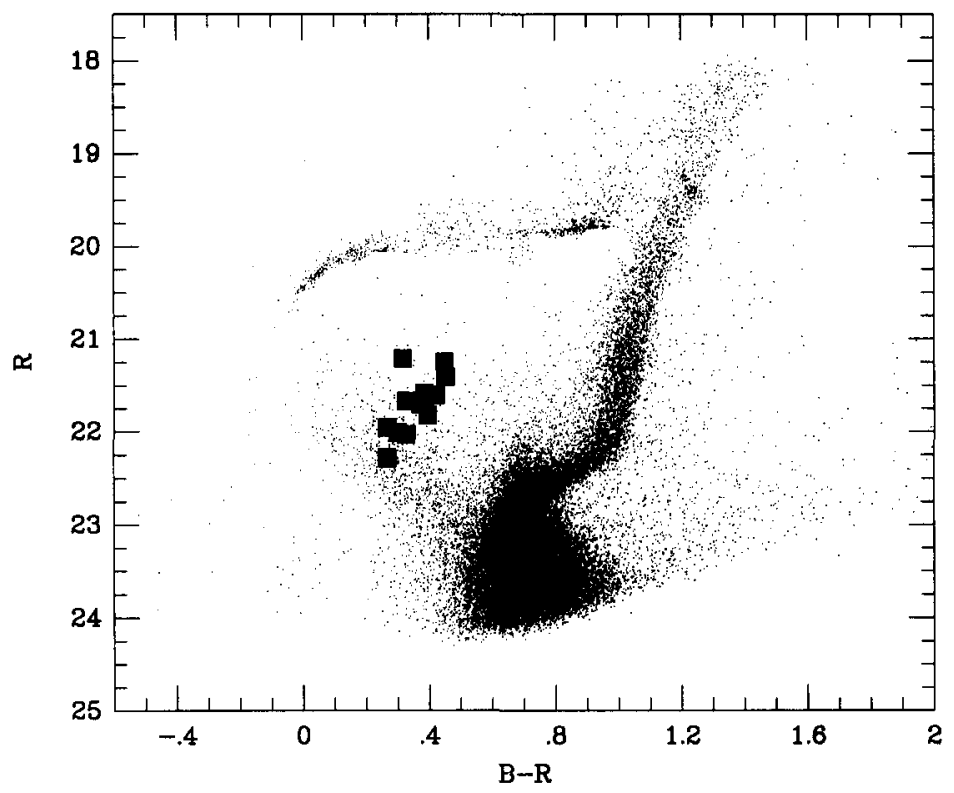

Figure 1. A color-magnitude diagram for the Sculptor dSph galaxy showing the locations of the dwarf Cepheids. Note how the distribution of these stars points directly to the RR Lyrae instability strip. However, unlike other pulsators, it is clear that most stars in the DC instability strip are not variable.

ods can be as short as about $1 \mathrm{hr}$. Thus, time-resolved photometric observations must be limited to very short exposure times to avoid undersampling the light curve, especially at phases of rapid brightness variations. Telescopes of $4-\mathrm{m}$ class with wide-field, high-sensitivity CCD cameras have proven up to this particular task. Not only Carina, but now Sculptor (Mateo, Hurley-Keller, \& Grebel 2000; Figure 1) and Fornax have been found to contain DCs.

\section{Future Prospects for Pulsating Variables in Local Group Dwarfs}

Concerted studies of the time-variability of stars within nearby dwarfs will help provide complete surveys that can serve as an important basis for comparison of the stellar populations of nearby dwarfs. As noted, 4-m telescopes and wide-field CCD mosaics are well-suited for this work. Studies for DCs can be carried out in short runs, but extensive surveys will require longer observations. In the era of 8 -m telescopes, it may become more practical to obtain the long runs needed to survey nearby systems in this manner. Ultimately these surveys can address issues such as (a) comparisons of the distance scales for all classes of pulsating variables, (b) measurements of the frequencies of different types of variables as a function of galaxy properties such as luminosity, mean metallicity, age distri- 
bution, etc., (c) definitive studies of the differences in the Fourier components of stars with different pulsation modes (which are easy to separate when the distances are the same), (d) the increased use of pulsators as precision probes of the depth of nearby galaxies, and (e) the use of these stars as tracers of the outer structure and shapes of dwarf galaxies as they are affected by tidal forces from the Milky Way and M31.

\section{References}

Alard, C. 1996, ApJ, 458, L17

Bono, G., Caputo, F., Santolamazza, P., Cassisi, S., \& Piersimoni, A. 1997, AJ, 113,2209

Grebel, E. K. 1997, Rev Mod Astron., 10, 29

Hubble, E. P. 1925, ApJ, 62, 409

Hurley-Keller, D., Mateo, M., \& Nemec, J. 1998, AJ, 115, 1840

Hurley-Keller, D., Mateo, M., \& Grebel, E. 1999, ApJ, 523, L25

Ibata, R. A., Gilmore, G., \& Irwin, M. J. 1994, Nature, 370, 194

Lepri, S., Mateo, M., Layden, A., Lemley, S., Olszewski, E., \& Morrison, H. 1997, BAAS, 29, 1341

Mateo, M. 1998, ARAA, 36, 435

Mateo, M., Fischer, P., \& Krzemiński, W. 1995, AJ, 110, 2166

Mateo, M., Hurley-Keller, D., \& Nemec, J. 1998, AJ, 115, 1856

Mateo, M., Hurley-Keller, D., \& Grebel, E. 2000, in preparation

Mateo, M., Kubiak, M., Szymanski, M., Kaluzny, J., Krzeminski, W., \& Udalski, A. 1995, AJ, 110, 1141

McNamara, D. 1997, PASP, 109, 1221

Nemec, J. M., Nemec, A. F. L., \& Lutz, T. E. 1994, AJ, 108, 222

Olszewski, E. W., Suntzeff, N. B., \& Mateo, M. 1996, ARAA, 34, 511

Poretti, E. 1999, A\&A, 343, 385

Skillman, E., Kennicutt, R. C., \& Hodge, P. W. 1989, ApJ, 347, 875

van den Bergh, S. 1999, A\&A Rev., 9, 273 